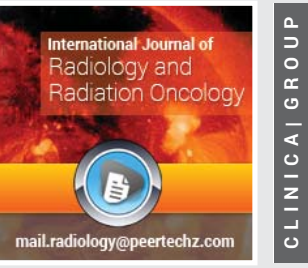

\section{Denaro Nerina*}

Medical Oncology Department, Santa Croce and Carle General Hospital Cuneo, Italy

Received: 29 June, 2018

Accepted: 21 August, 2018

Published: 22 August, 2018

*Corresponding author: Denaro Nerina, Medical Oncology Department, Santa Croce and Carle General Hospital Cuneo, Italy,

E-mail: nerinadenaro@hotmail.com

https://www.peertechz.com

Emerging data that radiotherapy can potentially convert the patient's own tumor into an in situ vaccine have raised significant interest for testing radiation in combination with immunotherapy. Moreover, the immune responses to localized irradiation may be the mediator of systemic effects (called the abscopal effect).

The rapid growth of clinical investigations combining immunotherapy and radiotherapy (RT), in the absence of rigorous hypothesis-driven trial design, may fail to truly exploit the emerging new paradigms, which could impair further development of the field. With novel immunotherapies entering clinical practice at an accelerating pace the need to understand how to best integrate radiation biology with tumor immunology to provide a solid rationale for clinical translation remains urgent [1].

Most of RT effects - cell death, inflammation, fibrosisare immunologically mediated.

Cell death occurs on a spectrum from immunogenic to tolerogenic. Radiotherapy has been shown to trigger the immune response by (a) induction of immunogenic cell death (ICD) broadening up the immune repertoire of $\mathrm{T}$ cells, (b) recruitment of $\mathrm{T}$ cells towards the irradiated tumour and (c) increasing vulnerability towards T-cell-mediated cell killing. Immunotherapy on the one hand may exert a direct antitumoral effect, modulating immune response especially in those hot tumours, on the other hand it may work as radiosensitizer also increasing effect and adverse events. Immune response correlated with significant pre-treatment tumor lymphocytic infiltration within such infiltrated tumors, referred as "hot", immune checkpoint inhibitors rescue anti-tumor $\mathrm{T}$ cells activity also in monotherapy with long lasting response, but in solid tumors only $20 \%$ of patients present these behaviours.

Radiotherapy modifies the tumor cell phenotype and the tumor microenvironment modulating the immune system, mainly in an switch-on modality.

Therefore, combining immunotherapy and radiotherapy looks very interesting.

There are more than 100 phase II and III trials on going combining radiotherapy and immunotherapies (both anti and programmed cell death protein 1 (PD-1) anti PD1 /PD ligand 1 (PD-L1) -Nivolumab, Pembrolizumab Durvalumab and antiCytotoxic T lymphocyte antigen 4 (CTLA-4) (Tremelimumab Ipilimumab).

DeMaria S, et al [1], suggested few rules to define the real advantage of combination by collecting data on a nation-wide registry of abscopal effects in patients who receive radiotherapy during treatment with immune checkpoint inhibitors (ICI); by expanding the use of pre-operative trials (pre-surgical and neoadjuvant) to study the irradiated tumor site and by investigating blood surrogate biomarkers of radiation-induced effects in tumor microenvironment (TME) in an international project.

It is not known which sequence of the applications of RT and immunotherapy is preferable, neither which is the dose and fractionation more effective. While lower single doses may impact on angiogenesis and immune cell infiltration, higher doses may impact on intra-tumoral induction (through the release of immunogenic cancer cell death) and production of type I interferons.

The therapeutic dose is established to destroy solid cancer limiting damage on normal tissue, but some preclinical study noted that repeatedly fraction is easier cause abscopal effect than high dosage radiotherapy, so could we use more hypofractionated radiation therapy to cure patient especially in population who want to use immunotherapy instead of conventional fractionation? This observation deserves further confirmation. 
Dovedi et al investigated in mouse models the benefit of concurrent anti PDL1 therapy versus sequential, achieving the best outcome with concomitant treatment, however Antonia et al showed amazing results in locally advanced non-small cell lung cancer treated with adjuvant Durvalumab $[2,3]$.

Moreover poly-immunotherapy appear in mouse models to obtain the highest response $[4,5]$.

The authors explained the reasons of this behaviour showing that radioresistant tumours presented higher levels of PD-L1 and persistent $\mathrm{T}$-cell exhaustion, with rapidly progressing disease. Twyman Saint-Victor et al. show that RT enhances the diversity of the T-cell receptor repertoire intratumorally (TCR) while anti-CTLA4 promotes expansion of effector T-cells and inhibits T-regulatory cells (Treg cells), and anti-PD-L1 reverses T-cell exhaustion. Similarly, these results obtained in mouse models were confirmed in melanoma patients [5].

The most important question is that RT and checkpoint inhibitors activate the immune system through two nonoverlapping mechanisms that make clinical synergy so exciting [5].

Table 1 summarizes the major unanswered questions among timing, doses, combinations and setting.

In conclusion much has been written recently about the immunostimulatory effects of radiation, but several mechanisms are not completely understood.

Different immunity in population means diverse genetic background, so gene sequencing may become good biomarkers just like tumor mutation burden, and some special gene like $\mathrm{MDM} 2 / 4$ could predict the efficacy of immunotherapy. Meanwhile, how to use gene sequencing to predict the efficacy and host's immunity stay unclear. Molecular findings highlight the role of DNA sensors and activators of innate. In murine models involving exposure to high-dose RT, innate immune signalling pathways activated by DNA damage have been shown to proceed through cytosolic DNA sensors. For example AIM2 (absent in melanoma 2) is a cytoplasmic DNA sensor

Table 1: Unanswered questions on the immune-radiotherapy

\begin{tabular}{|c|c|c|}
\hline \multicolumn{3}{|c|}{ Uncertainties } \\
\hline Radiotherapy dose & Daily fraction? & Total dose? \\
\hline Integration & Concurrent Immunotherapy? & Sequential immunotherapy? \\
\hline Agent & Anti PD1? Anti CTLA4? & Single agent? Polytreatment? \\
\hline Chemotherapy & Induction? & Consolidation? \\
\hline Setting & Neoadjuvant?Adjuvant? & Oligometastatic disease \\
\hline
\end{tabular}

that recognizes double-stranded DNA damaged by radiation. It induces proinflammatory cytokines and caspases. The cGAS (cyclic GMP-AMP synthase) is another cytosolic DNA sensor, which recognizes damaged DNA and activates STING (stimulator of interferon genes). In the presence of a checkpoint inhibitor and a radiotherapy regimen of $8 \mathrm{~Gy} \times 3$, cGAS (cyclic GMPAMP synthase) activating STING, induces the recruitment of dendritic cells, and the priming of antitumoral CD8 + T cells.

Therefore, the future biomarkers will be the combination of gene signature with microenvironment (including microbiome) information. There is an urgent need to develop guidelines and biomarkers for immune monitoring in trials testing radiation and immunotherapy.

In order to maximize the benefit achieved by $\mathrm{Rt}$ and immunotherapy, we need to use all rational combinations and sequences; in this way chemotherapy followed by RT plus immunotherapy may reach important results. Several chemotherapeutic drugs have immunostimulant properties. These studies need very clever programming and monitoring as if on the one hand Poly-treatment is a good way to get higher clinical remission rate, on the other hand it increases serious side effects.

Randomized trials to establish how to best induce abscopal effect in patients with metastatic disease are warranted. Future translational investigation will be the key for rational patient selection and optimal timing of immune modulation.

\section{References}

1. Coleman CN, Formenti SC (2016) Radiotherapy: Changing the Game in Immunotherapy. Trends Cancer 2: 286-294. Link: https://tinyurl.com/yd6qck8m

2. Dovedi SJ, Adlard AL, Lipowska-Bhalla G, McKenna C, Jones S, et al. (2014) Acquired resistance to fractionated radiotherapy can be overcome by concurrent PD-L1 blockade. Cancer Res 74: 5458-5468. Link: https://tinyurl.com/y9bvl4o7

3. Antonia SJ, Villegas A, Daniel D, Vicente D, Murakami S, et al. (2017) PACIFIC Investigators. Durvalumab after Chemoradiotherapy in Stage III Non-Small-Cell Lung Cancer. N Engl J Med 377: 1919-1929. Link: https://tinyurl.com/ycq9a7et

4. Wang $Y$, Deng W, Li N, Neri S, Sharma A, et al. (2018) Combining Immunotherapy and Radiotherapy for Cancer Treatment: Current Challenges and Future Directions. Front Pharmacol 9: 185. Link: https://tinyurl.com/y9ljgmwk

5. Twyman-Saint Victor C, Rech AJ, Maity A, Rengan R, Pauken KE, et al. (2015) Radiation and dual checkpoint blockade activate nonredundant immune mechanisms in cancer. Nature 520: 373-377. Link: https://tinyurl.com/y7ow5a3q

Copyright: (c) 2018 Nerina D. This is an open-access article distributed under the terms of the Creative Commons Attribution License, which permits unrestricted use, distribution, and $\mathrm{r}$ eproduction in any medium, provided the original author and source are credited. 